- zooxanthellae, which have a symbiotic relationship with reef-building corals. The algae provide the corals with energy and nutrients from photosynthesis; without them, the corals often die.

But to gauge the full extent of heat damage, Hughes's team conducted morecomprehensive underwater surveys of coral mortality, both at the peak of the observed bleaching in March and April, and again eight months later.

Many corals - especially those in the northern third of the reef - died immediately from heat stress. Others were killed more slowly, after their algal partners were expelled. The composition of coral assemblages on hundreds of individual reefs changed radically within just a few months of the heatwave. On severely bleached reefs, fast-growing coral species - which have complex shapes that provide important habitats - were replaced by slower-growing groups that shelter a lessdiverse community.

"The study paints a bleak picture of the sheer extent of coral loss on the Great Barrier Reef," says Nick Graham, a marine ecologist at Lancaster University, UK. Approximately onethird of the world's coral reefs were affected by bleaching in 2016. On the Great Barrier Reef, less than $10 \%$ of reefs escaped with no bleaching (see 'Bleached reef'), compared with more than $40 \%$ in previous bleaching events studied.

"It is now critical to understand how governance and local management can maximize recovery between recurrent heatwaves," Graham says.

Tim McClanahan, a conservation zoologist

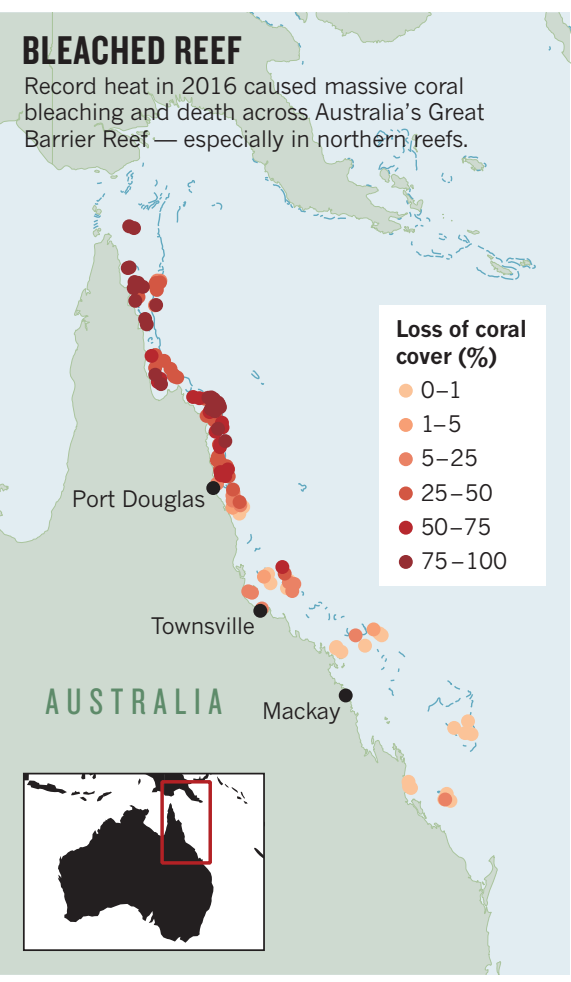

at the Wildlife Conservation Society in Mombasa, Kenya, says the study's findings might not predict how other reefs will cope with a warmer world. Responses might depend on the corals' life histories and local environmental conditions. "Global warming will result in more heat-stress events," he says, but "there is accumulating evidence that corals do acclimate".
Before the extreme 2016 incident, global coral bleaching had been observed just twice: in 1998 and 2002. Coral colonies can recover from such events, especially given ò that the species most susceptible to dying from heat stress are among the fastest-growing corals. But harmful warming events are occurring more frequently, and scientists think that full recovery is becoming increasingly difficult ${ }^{2}$.

Researchers have also found that local protection of reefs and surrounding waters does little to make corals less sensitive to heat ${ }^{3}$. Rather, global changes such as ocean acidification might further increase environmental stress.

The fate of tropical coral reefs - including the iconic Great Barrier Reef - therefore depends on efforts to mitigate climate change, says Graham. "A future with coral reefs, their rich diversity and the livelihoods they provide to people is quite simple. It will only be possible if carbon emissions are rapidly reduced," he says.

But even if that happens, tomorrow's reefs might look different from today's, as the mix of species changes in favour of those that can best cope with inevitable climate change, says Hughes. "This transition is already under way, faster than many of us expected," he says. "The Great Barrier is shifting radically, a trend that will continue for the next century or more." -

\footnotetext{
1. Hughes, T. P. et al. Nature https://doi.org/10.1038/ s41586-018-0041-2 (2018).

2. Hughes, T. P. et al. Science $359,80-83$ (2018).

3. Hughes, T. P. et al. Nature 543, 373-377 (2017).
}

\title{
East Asia braces for surge in deadly tick-borne virus
}

\section{Rapid rise in number of infections concerns researchers.}

\section{BY DAVID CYRANOSKI}

$\mathrm{I}$ nfectious-disease experts in East Asia are preparing for this year's wave of a lethal tick-borne virus. The virus causes a disease called severe fever with thrombocytopenia syndrome (SFTS), which has affected a rapidly growing number of people since it emerged nearly a decade ago.

Scientists in the region say they are worried by the rising incidence of the disease, and by signs that the virus can spread more easily than previously thought. In March, Japan launched the first clinical trial of a drug to treat the infection, and some researchers say that governments should devote more resources to raising awareness and studying the virus.

"It is our responsibility to come up with an effective treatment," says Masayuki Saijo, a virologist at the National Institute of Infectious Diseases in Tokyo, who helped to launch the trial.

Cases of SFTS were first reported in China in 2009 (X.-J. Yu et al. N. Engl. J. Med. 364, 1523$1532 ; 2011)$. Researchers identified the virus responsible in blood samples from a cluster of people who shared a combination of symptoms, including high fever, gastrointestinal problems, low white blood cell count and low platelet count (thrombocytopenia).

The virus killed $30 \%$ of those infected in China that year. It was even more lethal when the first cases appeared in Japan and South Korea in 2013. More than one-third of those infected in Japan and nearly half of those infected in South Korea died that year.

And the number of cases in each country has risen sharply. In 2013, there were 36 reported cases in South Korea; by 2017, the number had jumped to 270. In 2010, China reported 71 cases; in 2016, there were around 2,600. Japan experienced a 50\% 


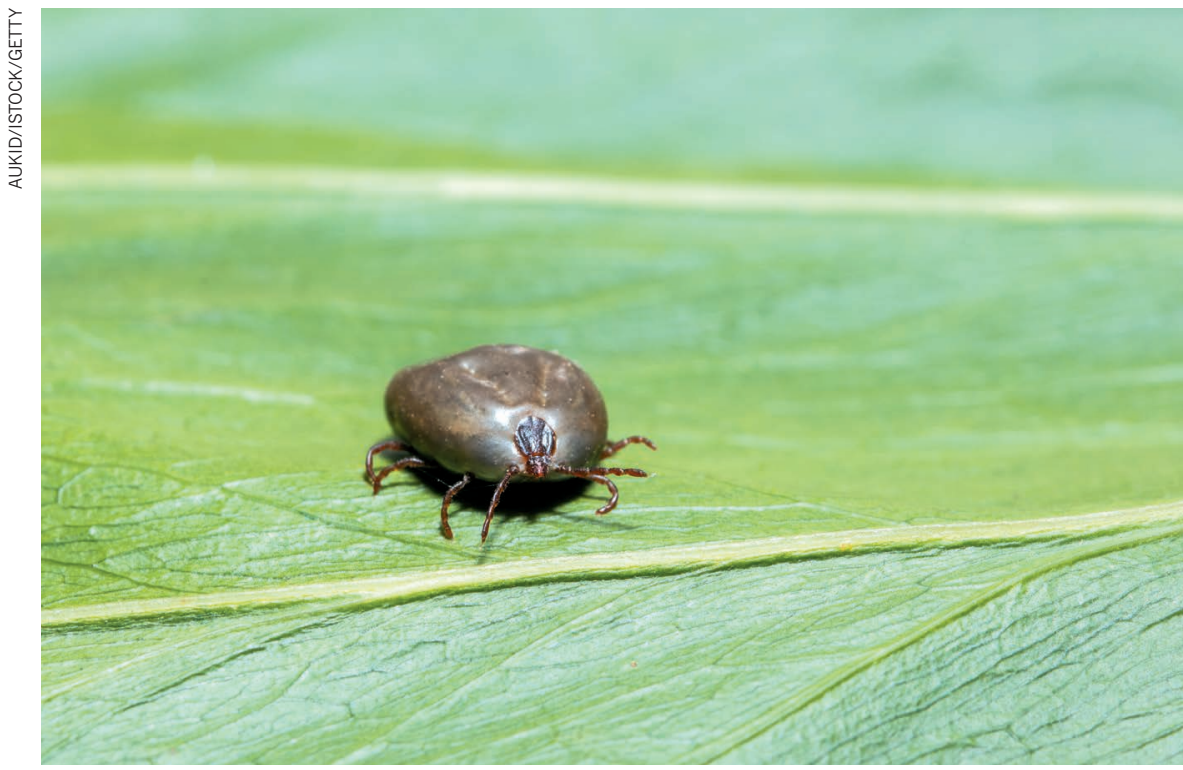

The tick Haemaphysalis longicornis transmits an emerging, potentially fatal virus to people.

increase between 2016 and 2017.

All three countries have implemented measures aimed at educating local physicians and citizens in endemic areas about the risks of tick bites. Those infected now fare much better. In China, only around 3\% of people infected died in 2016, and in Japan the number fell to $8 \%$. In South Korea, the figure dropped from $47 \%$ in 2013 to $20 \%$ in 2017. Scientists credit the reduced fatality to earlier recognition and better general treatment - although no cure exists - and to the likelihood that wider surveillance has led physicians to recognize mild as well as severe cases.

The SFTS virus is not expected to evolve into a rapidly transmitted disease like Ebola. And infections are generally limited to people, such as farmers or hunters, who come

\section{"It is our responsibility to come up with an effective treatment."} into contact with the animals that carry Haemaphysalis longicornis, the tick that harbours the virus.

But many say that the virus's toll and potential threat have been under-appreciated. Those infected have a better prognosis, but the virus still kills a higher percentage than any other infectious disease in South Korea, says Keun-Hwa Lee, a microbiologist at Jeju National University in South Korea. And the higher number of infections means that the disease claims more than 100 lives globally each year.

Many animals, including goats, cattle, sheep and deer, expose humans to the ticks, and are often infected without showing symptoms. Current control efforts that focus on known endemic areas could fail, says Bao Chang-jun, a biostatistician at Jiangsu Provincial Center for Disease Control and Prevention in Nanjing. The course of the epidemic "may change with human activities and climate change", says Bao. "It's necessary to conduct research on potential risk areas."

Two reports from Japanese health officials last year caused particular alarm. One stated that a woman had been infected through a cat bite, and the other that a man had been infected by his dog. "To the warnings of previous years, we have to add the risk of touching sick domestic animals," says Kazunori Oishi, director of the Infectious Disease Surveillance Center in Tokyo.

\section{CLINICAL TRIAL}

Last month, Japan began a clinical trial of an influenza drug, favipiravir, that was used to treat Ebola during the 2014 outbreak in West Africa. The drug is effective on viruses with a certain molecular structure that Ebola and SFTS share, says Saijo.

Although the number of cases has risen sharply, scientists can't say whether the increase is due to heightened surveillance and awareness, a real growth in the number of ticks and the animals that carry them, or an increase in risk as humans encroach on areas where the disease is endemic. Shigeru Morikawa, director of the department of veterinary science at Japan's National Institute of Infectious Diseases, says that some researchers suspect the number of ticks has increased because fewer people hunt wild animals in Japan now, and this has allowed deer and boar populations to surge.

Researchers say they have many questions about the virus and how it spreads, but they suspect that the chances to study the disease will go up soon, as warm weather returns and people flock to the outdoors, where they can come into contact with the ticks. "There will be more cases," says Hideki Hasegawa, a pathologist at the National Institute of Infectious Diseases. "The season is just beginning."

\section{CLIMATE CHANGE}

\section{Big prize for methane probe}

\author{
US green group wins \\ millions to launch satellite.
}

\section{BY JEFF TOLLEFSON}

A n environmental group in the United States has been awarded tens of millions of dollars to develop a satellite to help track emissions of the greenhouse gas methane from oil and gas facilities around the world.

If the Environmental Defense Fund (EDF) succeeds in launching its probe, it could be the first environmental organization to send its own satellite into space. Its work is being funded through the Audacious Project, a joint effort of the non-profit group TED and philanthropic organizations such as the Bill \& Melinda Gates Foundation.

The EDF, based in New York City, aims to launch 'MethaneSAT' as early as 2020, with the help of scientific partners at Harvard University and the Harvard-Smithsonian Center for Astrophysics in Cambridge, Massachusetts. The group says that the probe will make the most-precise measurements yet of methane from space, and its data will be freely available.

The oil and gas industry emits around 76 million tonnes of methane globally each year, according to the International Energy Agency in Paris. That's enough to power about 285 million US homes. The EDF's goal is to monitor emissions from roughly 50 sites that account for around $80 \%$ of the world's oil and gas production. But the satellite could also be used to estimate emissions from landfills and agriculture. "We need good solid data so that we really can support global action on climate change, and we've got to do it fast," says Steven Hamburg, the EDF's chief scientist.

The most-detailed measurements of atmospheric methane concentrations available come from the European Space Agency's Sentinel$5 \mathrm{P}$ spacecraft, which was launched in October 2017. It provides global coverage at a resolution of nearly 50 square kilometres.

The EDF team is designing MethaneSAT to provide measurements at a resolution of 1 square kilometre, with global coverage at least once a week. That information can then be plugged into atmospheric models to calculate cumulative emissions across larger areas, says Steve Wofsy, an atmospheric scientist at Harvard who is working on the project.

"EDF has a very good team, and I have no doubt that it can be done," says Charles Elachi, who formerly headed NASA's Jet Propulsion Laboratory in Pasadena, California. "The challenge is how much it's going to cost." - 